\title{
Transcriptomic Analysis of MicroRNA Expression in
}

\section{Enamel-Producing Cells}

\author{
Minou Nirvani $^{1 *}$, Cuong Khuu ${ }^{1}$, Amela Tulek ${ }^{1}$, Tor Paaske Utheim ${ }^{1,2,3}$, \\ Lars Peter Sand ${ }^{1}$, Malcolm L. Snead ${ }^{4}$ and Amer Sehic ${ }^{1,3}$ \\ ${ }^{1}$ Department of Oral Biology, Faculty of Dentistry, University of Oslo, Oslo, Norway \\ ${ }^{2}$ Department of Medical Biochemistry, Oslo University Hospital, Oslo, Norway \\ ${ }^{3}$ Department of Maxillofacial Surgery, Oslo University Hospital, Oslo, Norway \\ ${ }^{4}$ Center for Craniofacial Molecular Biology, Herman Ostrow School of Dentistry, \\ University of Southern California, Los Angeles, CA, USA
}

* Corresponding author:

Minou Nirvani,

Department of Oral Biology, University of Oslo,

Box 1052 Blindern, 0316 Oslo, Norway

Telephone: +4722840343

E-mail: minou.nirvani@ odont.uio.no

\section{Short title:}

MicroRNAs in ameloblast-like cell line LS8 


\section{Abstract}

There is little evidence for the involvement of microRNAs (miRNAs) in the regulation of circadian rhythms during enamel development. Few studies have used ameloblast-like cell line LS8 to study the circadian rhythm of gene activities related to enamel formation. However, the transcriptomic analysis of miRNA expression in LS8 cells have not been established yet. In this study, we analyze the oscillations of miRNAs in LS8 cells during one-day cycle of 24 hours by next generation deep sequencing. After removal of low quality reads, contaminants, and ligation products, we obtained a high number of clean reads in all 12 samples from four different time points. The length distribution analysis indicated that $77.5 \%$ of clean reads were between 21 and 24 nucleotides (nt), of which $35.81 \%$ reads exhibited a length of $22 \mathrm{nt}$. In total, we identified 1471 miRNAs in LS8 cells throughout all four time-points. 1330 (90.41\%) miRNAs were identified as known miRNA sequences, whereas 139 (9.59\%) were unannotated and classified as novel miRNA sequences. The differential expression analysis showed that 191 known miRNAs exhibited significantly $(\mathrm{P}$-value $<0.01)$ different levels of expression across three time-points investigated (T6, T12, and T18) compared to T0. Verification of sequencing data using qRT-PCR on six selected miRNAs suggested good correlation between the two methods. Gene Ontology (GO) and Kyoto Encyclopedia of Genes and Genomes (KEGG) pathway analysis revealed significant enrichment of predicted target genes of differentially expressed miRNAs. The present study shows that miRNAs are highly expressed in LS8 cells and that a significant number of them oscillate during one-day cycle of 24 hours. This is the first transcriptomic analysis of miRNAs in ameloblast-like cell line LS8 that can be potentially used to further characterize the epigenetic regulation of miRNAs during enamel formation.

Keywords: Ameloblasts, Dental enamel, LS8 cells, MicroRNAs, Transcriptome profiling 


\section{Introduction}

Dental enamel is the most mineralized tissue in vertebrates. Formation of enamel starts at the enamel-dentine junction and continues outward by layered apposition of the matrix produced and secreted by the ameloblast cells. The movement of the ameloblasts as a whole is mirrored by the incremental lines of enamel, the Retzius lines, which may evidence physiological or pathological events affecting enamel formation $(1,2)$. We have recently showed that incremental lines are evident in mouse molar enamel, and that they probably represent a daily rhythm in enamel production (3).

Circadian rhythms in mammals are driven by an endogenous timekeeping system consisting of the body`s central pacemaker situated in the suprachiasmatic nucleus of the hypothalamus and subordinate clock genes in peripheral tissue. The circadian clock continuously adapts and is driven by cyclic events (4). It is known that the circadian clock regulates several physiological processes (5), including that of other mineralized tissue $(6,7)$. Therefore, it is reasonable to assume that the observed incremental markings in dental enamel are controlled by the circadian clock. A recent investigation, comparative gene-expression profiling of the mouse molar tooth germ, revealed high expression of microRNAs (miRNAs) and oscillation in a circadian pattern in the tooth germ (8). The computational prediction suggested that the circadian expression of specific miRNAs was correlated with the expression of corresponding target mRNA (8). MiRNAs are small ( 21 nucleotides) non-coding RNA molecules that are expressed endogenously and regulate gene expression at a posttranscriptional level $(9,10)$. It has been shown that miRNAs exhibit important functions in modulating clock genes (11), and that they are highly expressed during tooth development (12-15). However, their exact role in the interplay between circadian clock and enamel development remains elusive.

As enamel formation begins a cytoplasmic outgrowth called the Tomes' process, 
protrudes from the distal surface of each ameloblast (16). The Tomes' process does not entail the whole distal surface of the cell, but leaves a rim of the original distal surface at the base of the process. This surface, including the Tomes' process, forms the secretory surface of the ameloblast, being responsible for secretion of enamel matrix proteins (16). Once the maturation phase commences, the Tomes' process is gradually lost, and the secretion of enamel matrix proteins ends. The mouse immortalized ameloblast-derived cell line, LS8, was originally derived by Chen and colleagues from the enamel organ epithelial cells isolated from the first mouse molar at embryonic day 16 (17). LS8 cells exhibit a quasi-Tomes' process (17), express high levels of transcripts related to secretory-stage activities, i.e. Amelx, Ambn, Enam, and Mmp20 (18), and therefore are characterized as secretory phase ameloblasts. Although LS8 cells show high levels of expression of several genes associated with enamel formation, they do not produce a typical rodent extracellular enamel matrix in culture $(19,20)$.

LS8 cells have previously been used for the investigation of amelogenin (21-24) and ameloblastin $(25,26)$ gene expression in vitro, and for the study of mechanisms for fluoride uptake and endoplasmic reticulum related stress activities (27). Lacruz and collaborates have used LS8 cell line to study circadian rhythm of gene activities related to enamel formation (28). Their findings indicated that the essential genetic regulators of the circadian clock are expressed in LS8 cell cultures, and that their expression profile follows a circadian pattern. Furthermore, the expression and function of specific miRNAs has been studied in LS8 cells, e.g. miR-153 $(29,30)$ and miR-31 (29), suggesting miRNAs to be involved in regulation of key processes during amelogenesis. However, the genome-wide expression of miRNAs in LS8 cells have so far not been investigated. The aim of the present study was to analyze the expression levels of miRNAs in LS8 cells by deep sequencing, and to investigate whether their expression oscillate during one-day cycle of 24 hours. 


\section{Methods}

\subsection{Cell Culture and Treatment}

The LS8 cell line was maintained in Dulbecco's modified Eagle's medium (DMEM) (SigmaAldrich, St Louis, MO, USA) supplemented with 10\% fetal bovine serum, 2 mML-glutamine, $1 \%(\mathrm{v} / \mathrm{v})$ penicillin, $1 \%(\mathrm{v} / \mathrm{v})$ streptomycin, and $0.25 \mu \mathrm{g} / \mathrm{ml}$ amphotericin B (all reagents purchased from Lonza, Basel, Switzerland). The cells were cultured at $37^{\circ} \mathrm{C}$ and passaged twice a week at $80 \%$ confluence. In order to induce cell cycle synchronization and for measuring the circadian effects of miRNA expression, LS8 cells were exposed for 2 hours to serum-free medium containing $20 \mathrm{mM}$ forskolin. After 2 hours, forskolin containing medium was replaced with regular culture medium DMEM. As previously described, forskolin is known to induce cell cycle synchronization of cultured cells (31). Triplets of samples were harvested at four time-points; first 2 hours after ended forskolin treatment (T0), and then repeated every 6 hours, i.e. at T6, T12, and T18.

\subsection{MicroRNA Extraction and Library Preparation}

Harvested cells were lysed and miRNAs were extracted using mirPremier (Sigma-Aldrich, St Louis, MO, USA) as described by the manufacturer. This yielded RNA fractions exhibiting ratios of absorbance $(\mathrm{A})_{260} / \mathrm{A}_{280}$ and $\mathrm{A}_{260} / \mathrm{A}_{230}$ of at least 1.8 and 2.0, respectively. The concentrations of solutions of purified RNA were assayed using Nanodrop ND-1000 spectrophotometer (Thermo Scientific, Waltham, MA, USA). RNA degradation and contamination were monitored on $1 \%$ agarose gels. RNA purity was checked using the NanoPhotometer spectrophotometer (IMPLEN, CA, USA). RNA integrity was assessed using the RNA Nano 6000 Assay Kit of the Agilent Bioanalyzer 2100 system (Agilent Technologies, CA, USA). 
A total amount of $1 \mu \mathrm{g}$ miRNA per sample was used as input material for the small RNA library. Sequencing libraries were generated using NEBNext Multiplex Small RNA Library Prep Set for Illumina (NEB, USA) following manufacturer's recommendations, and index codes were added to attribute sequences to each sample. Briefly, NEB 3' SR Adaptor was directly and specifically ligated to 3' end of the miRNA. After the 3' ligation reaction, the SR RT Primer hybridized to the excess of 3' SR Adaptor (that remained free after the 3' ligation reaction) and transformed the single-stranded DNA adaptor into a double-stranded DNA molecule. The $5^{\prime}$ end adapter was ligated to $5^{\prime}$ ends of miRNAs. Then, first strand cDNA was synthesized using M-MuLV Reverse Transcriptase (RNase H-). PCR amplification was performed using LongAmp Taq 2X Master Mix, SR Primer for Illumina and index (X) primer. PCR products were purified on $8 \%$ polyacrylamide gel (100V, $80 \mathrm{~min})$. DNA fragments corresponding to 140 160bp (the length of small noncoding RNA plus the 3' and 5' adaptors) were recovered and dissolved in $8 \mu \mathrm{L}$ elution buffer. At last, library quality was assessed on the Agilent Bioanalyzer 2100 system using DNA High Sensitivity Chips. The clustering of the index-coded samples was performed on a cBot Cluster Generation System using TruSeq SR Cluster Kit v3-cBot-HS (Illumina) according to the manufacturer's instructions.

\subsection{MicroRNA Sequencing and Data Analysis}

After cluster generation, twelve single-end miRNA libraries were sequenced by Novogene Bioinformatics Technology Co. Ltd. (Beijing, China) on Illumina HiSeq 2500/2000 platform. The clean reads were obtained by mapping the reads to RepeatMasker after adapter sequences, reads containing poly $\mathrm{A}$ or $\mathrm{T}$ or $\mathrm{G}$ or $\mathrm{C}$ and low-quality reads had been removed from the raw data. To identify known murine miRNAs from the clean reads, miRBase v. 21 was used as a reference (32), and miREvo (33) and mirdeep2 (34) were used for prediction of novel miRNAs based on the characteristics of hairpin structure of the miRNA precursor. 
The miRNA expression levels were estimated by TPM (Transcript Per Million = miRNA total reads/total clean reads) values. Differential expression of miRNAs between two groups was performed using the DEGseq R Package. The P-value was adjusted using q-values.

Q-value $<0.01$ and $\mid \log 2$ (fold change) $\mid>1$ was set as the threshold for significantly differential expression by default (35). Prediction of target genes of differentially expressed miRNAs was performed by psRobot_tar in miRanda (36). Gene Ontology (GO) enrichment analysis using GOseq was applied on target gene candidates of differentially expressed miRNAs (37). KOBAS software (38) was used in KEGG pathway (39) to test the statistical enrichment of differentially expressed candidates for their roles in biological system.

\subsection{Validation of MicroRNA-Sequenced Data with Quantitative Real-Time PCR}

TaqMan Advanced miRNA assays (Applied Biosystems, Forster City, CA, USA) was used to validate miRNA-sequenced results of mmu-miR-423-3p, mmu-miR-218-5p, mmu-miR-2105p, mmu-miR-152-5p, mmu-miR-29b-1-5p, mmu-miR-21a-5p, mmu-miR-181b-1-3p, and mmu-miR-16-5p. In brief, $5 \mathrm{ng}$ of total small RNA was used for cDNA synthesis. The assay uses 5' adapter ligation to mature miRNAs for generating cDNA for all miRNAs in a single reaction, and $5 \mu \mathrm{l}$ of the diluted cDNA reaction mix was used in each well with a total volume of $20 \mu \mathrm{l}$ per well. qRT-PCR reactions were performed in a 96-wells PCR reaction plate on AriaMx Real-Time PCR System (Agilent Technologies, Santa Clare, USA). Data were normalized to mmu-miR-423-3p. 


\section{Results}

\subsection{Sequence Analysis}

In order to characterize the expression of miRNAs in LS8 cells and to elucidate whether their expression profiles oscillate during one-day cycle of 24 hours, we conducted a comparative transcriptomic analysis among cell samples harvested at four time-points. Transcriptomic analysis was carried out in triplicate for all four time-points, and a total of twelve cDNA libraries, named T0 (A, B, C), T6 (A, B, C), T12 (A, B, C), and T18 (A, B, C) were constructed and sequenced (Supplementary material 1).

The number of raw reads obtained per sample ranged from $20,273,866$ to $23,283,386$ with a mean of 21,384,233 (Supplementary material 1). A quality score (Q-score) was predicted for all reads and indicates the probability of an error in base calling (40). The relationship between the base call quality scores and their corresponding error probabilities for all samples is presented in Supplementary material 1. Our results suggested Q20 (one predicted incorrect base call in 100) and Q30 (one predicted incorrect base call in 1,000) to be higher than $97.32 \%$ and $94.59 \%$, respectively. The content of $\mathrm{G}$ base and $\mathrm{C}$ base was determined, accounting for the overall GC content over 49.29\% (Supplementary material 1). Clean reads for small RNAs were acquired from raw reads by removing the reads with poly Ns $(\mathrm{N}>10 \%)$, reads with the 5'-adapter contaminants, reads without the 3 '-adapter or insert tags, and reads with poly A/T/G/C (Table 2). After removal of low-quality reads, contaminants, and ligation products, the number of clean reads obtained per sample ranged from $19,803,177$ to $22,397,187$ with a mean of 20,769,544 (Supplementary material 2). The clean reads were clustered into unique sequences, and the length analysis indicated that $77.5 \%$ of the length distributions of clean reads in all samples were identified to be between 21 and 24 nt, with $35.81 \%$ of reads exhibiting 22 
nt length (Figure 1). This is in accordance with typical characteristics of Dicer processing products (41).

\subsection{Detection of MicroRNAs in LS8 Cells}

In total, as shown in Table 1, we identified 1471 miRNAs across all time-points (T0, T6, T12, and T18) in LS8 cells. Furthermore, 1330 (90.41\%) miRNAs were identified as known miRNA sequences, whereas 139 (9.59\%) were unannotated and classified as novel miRNAs. At each time-point investigated, including all triplicate samples, about 1000 miRNAs were detected with approximately $91 \%$ known and 9\% novel miRNAs (Table 1).

\subsection{Differential Expression of Known MicroRNAs in LS8 Cells}

The differential expression analysis showed that 191 known miRNAs exhibited significantly (P-value $<0.01)$ different levels of expression across three time-points investigated (T6, T12, and T18) compared to T0 (Figure 2). At T6, compared to T0, 67 miRNAs were differentially expressed, 33 miRNAs being down-regulated and 34 up-regulated (Table 2). At T12, only 5 miRNAs were differentially expressed compared to T0, with 2 miRNAs being down-regulated and 3 up-regulated (Table 3). Finally, at T18, 156 miRNAs were differentially expressed, with 78 down-regulated and 78 up-regulated miRNAs (Table 4). In total, among the differentially expressed known miRNAs, 115 miRNAs were up-regulated and 113 down-regulated compared to T0 (Tables 2-4), demonstrating that some of the miRNAs were differentially expressed across two or three time-points (Figure 2). The overlapping combinations of differentially expressed miRNAs are presented in Figure 2. Interestingly, only one miRNA, mmu-miR-181b-1-3p, was detected as differentially expressed across all three time-points (Figure 2, Tables 2-4), however, the expression level was low. Compared to T0, mmu-miR-181b-1-3p was up-regulated at T6 and down-regulated at T12 and T18 (Tables 2-4). On the other hand, our results showed that 
33, 2, and 120 differentially expressed miRNAs were specific for T6, T12, and T18, respectively (Figure 2, Tables 2-4).

\subsection{Expression of Novel MicroRNAs in LS8 Cells}

In total, 139 novel miRNAs were detected across all time-points (Table 1). Among these, 94 exhibited low levels of expression (read count $<5$ across all time-points) and were excluded from further analysis. The remaining 45 novel miRNAs, including their sequences, are presented in Supplementary material 3. All miRNA sequences were detected at T0 and T12, however, only two of them, i.e. novel_1 and novel_3, were expressed at all time-points. Furthermore, at T6 two miRNAs (novel_1 and novel_3) and at T18 four miRNAs (novel_1, novel_3, novel_69, and novel_79) were detected (Supplementary material 3). Differential analysis confirmed that among all detected novel miRNA, only two (novel_15 and novel_144) exhibited significantly $(\mathrm{P}$-value $<0.01)$ different levels of expression, being up-regulated at T12 compared to T0 (Supplementary material 3).

\subsection{Verification of Transcriptomic Analysis with qRT-PCR}

In order to validate the reliability of the sequencing data, using mmu-miR-423-3p as normalization control, expression levels of seven differentially expressed miRNAs (mmu-miR29b-1-5p, mmu-miR-152-5p, mmu-miR-181b-1-3p, mmu-miR-210-5p, mmu-miR-16-5p, mmu-miR-21a-5p, and mmu-miR-218-5p) were verified by real-time PCR using miRNA specific primers. The real-time PCR results for each time-point, as an average of three triplicates, were in general in agreement with the results obtained from deep sequencing, following the same expression trends (Figure 3). Mmu-miR-181b-1-3p, however, exhibited low levels of expression and was not detectable with qPCR. 


\subsection{Functional Analysis of Differentially Expressed microRNAs}

PsRobot_tar in miRanda was used to predict the targets of differentially expressed miRNAs at all time-points investigated. In order to further characterize the function of these predicted genes, GO term and KEGG pathway were subsequently carried out. GO analysis was performed to study the biological processes, cellular components, and specific molecular functions of predicted targets that were associated with differentially expressed miRNAs. Our results suggested that differentially expressed miRNAs were associated with the genes enriched in numerous biological processes, e.g.: "binding", "protein binding", "ion binding", "organic cyclic compound binding", and "heterocyclic compound binding" (Figure 4). Similarly, the following cellular components were highly affected: "cellular component", “intracellular", "intracellular part", "organelle", and "intracellular organelle" (Figure 4), while the most affected molecular functions include: "metabolic process", "organic substance metabolic process", "primary metabolic process", "cellular metabolic process", and "macromolecule metabolic process" (Figure 4). Interestingly, the number of associated target genes was highly differing across the three time-points (T6, T12, and T18) when compared to T0 (Figure 4).

Pathway analysis was used to determine the involvement of predicted genes in biological system according to KEGG. Several KEGG pathways were significantly enriched by target genes of differentially expressed miRNAs, with the following pathways exhibiting most numerous associated genes: "pathways in cancer", "MAPK signaling pathway", “endocytosis”, "Ras signaling pathway", and "metabolic pathways" (Figure 5). The most striking finding from these results was that at $\mathrm{T} 18,1172$ predicted genes were highly associated with "metabolic pathways" (Figure 5c). 


\section{Discussion}

Possible functions and biological significance of miRNAs have been the subject of both experimental $(42)$ and theoretical $(43,44)$ studies. Although some miRNAs have been well studied, the majority of them and their roles in diverse biological processes still need to be completely understood. A report by Cheng et al. brought new insights into possible roles of miRNAs in modulating circadian clocks, revealing that the interplay between different types of RNAs and proteins can exert modulation of circadian-clock period and entrainment (42). Studies of the time courses of miRNA expression in developing murine molar tooth germ have shown these to be highly dynamic (14). A recent study also suggested that miRNAs modulate tooth morphogenesis and ameloblast differentiation, perhaps largely by fine-tuning of signaling networks (45).

The few available ameloblast-like cell lines that are under investigation by the research community include mouse LS8 (17) and ALC (46), rat HAT-7 (47) and SF2-24 (48), and porcine PABSo-E (49). LS8 (28) and HAT-7 cells (50) have been used to investigate circadian rhythm gene activities related to enamel formation, and the molecular profiles of only few selected enamel-specific genes have been carried out in LS8 and ALC cells (18). However, to the best of our knowledge, this is the first study that investigates the genome-wide expression of miRNAs in an ameloblast-like cell line. To elucidate the potential oscillating expression of miRNAs in LS8 cells, we have characterized the expression profile of miRNAs during one-day cycle of 24 hours. LS8 cells are immortalized with a SV40 T antigen (17), which can create some modulation of transcription factors due to binding resulting in over/under expression. However, when Lacruz and colleagues analyzed LS8 cells, they measured a $24 \mathrm{~h}$ 
cycle for amelogenin, solute pumps, and two of the principle positive and negative regulators of circadian cycles (28). They also identified E-boxes, potential binding sites for these regulators in the amelogenin and ameloblastin promoter regions, which they have had characterized. The cells showed the same cycle as developing molars from mice entrained to a light-dark cycle, therefore, it is reasonable to assume that the distortion of miRNAs in the present study is minimal.

Using deep sequencing, the present study aimed to systematically describe the oscillation of miRNAs in LS8 cells. Three technical replicates were used for each of four timepoints, resulting in a total of twelve cDNA libraries. Our results demonstrated good agreement between all replicates and a high number of clean reads was obtained in all samples. The most conspicuous findings emerging from our results is that as many as 1471 miRNAs were detected in LS8 cells across all time-points investigated (Table 1). A comparative analysis further demonstrated that 191 miRNAs exhibited significantly different levels of expression during one day cycle of 24 hours, suggesting these miRNAs to be important for the circadian molecular machinery of LS8 cells. Validation of deep sequencing data using RT-PCR suggested good correlation between the two methods as judged from results obtained with six miRNAs whose expression was monitored using both methods (Figure 3). Mmu-miR-181b-1-3p was not detectable with $\mathrm{qPCR}$, most probably due to low expression levels. It has previously been shown that the correlation between deep sequencing data and qPCR results is poor for low expressed miRNAs (51).

It is not surprising that a larger number of miRNAs was detected in LS8 cells in the present study using deep sequencing compared to previous microarray-based studies using a total of 728 probes recognizing rat and mouse miRNA sequences $(8,14)$. Using microarrays, miRNA expression profiles have been established at selected time-points (E15.5, P0, and P5) during development of the murine first mandibular molar tooth germ (14). The results obtained 
from this previous study showed that 76 miRNAs were expressed in molar tooth germ, with 68 miRNAs being expressed at all three time-points. Interestingly, when compared with the results from the present study, 38 of these differentially expressed miRNAs detected in molar tooth germ, were also found to exhibit significantly different levels of expression in LS8 cells during one day cycle of 24 hours. Recently, by carrying out comparative gene-expression profiling of the mouse molar tooth germ, we identified 32 miRNAs that were expressed in a circadian manner (8). Six of these, i.e. mmu-miR-145, mmu-miR-182, mmu-miR-210, mmu-miR-322, mmu-let-7b, and mmu-let-7d, were also differentially expressed in LS8 cells, as shown in the present study (Tables $2 \& 4$ ). One of these miRNAs, i.e. mmu-miR-182, is of particular interest since previous in vitro study provided evidence for that Clock is a direct target of miR-182 (52). Mmu-miR-182 was highly expressed and oscillated in a circadian pattern in the molar tooth germ, however, in addition, the same study showed that the expression of Clock was inversely correlated with the expression of mmu-miR-182 (8). Our results showed that mmu-miR-182 in LS8 cells was down-regulated at T6 and T18 compared to T0, providing an increasing amount of evidence supporting a general positive correlation between mmu-miR-182 and peripheral circadian regulation in ameloblasts. However, further functional analysis of mmu-miR-182 is warranted.

Previously, Yin and colleagues have conducted a genome-wide analysis of miRNA and mRNA transcriptomes in secretory- and maturation stage rat enamel organs (29). In this study they also used luciferase reporter assays in LS8 cells to provide evidence that miR-153 and miR-31 are potential regulators for their predicted target mRNAs Lampl and Tfrc, respectively. Before the transfection of miR-153 mimic, the expression of miR-153 was not detected (29), which is in accordance with our findings (results not shown). In the present study, mmu-miR181b-1-3p was the only miRNA detected as differentially expressed across all time-points investigated (Figure 2, Tables 2-4). However, mmu-miR-181b-1-3p was not detectable with 
qPCR. The function of mmu-miR-181b-1-3p in ameloblasts is uncertain, however, it has recently been identified as an important candidate for promoting epithelial-mesenchymal transition in breast cancer cells (53). The use of mimic in order to increase the expression levels of mmu-miR-181b-1-3p may be appropriate approach for further investigation of the role of miR-181b-1-3p in LS8 cells.

GO mapping was carried out to highlight the functions of differentially expressed miRNAs and grouped into three main domains: biological processes, molecular functions, and cellular components (Figure 4). The functional GO distribution demonstrated that several predicted genes involved in biological and metabolic processes may be influenced by oscillations of miRNAs. Although miRNAs represent only one of several mechanisms regulating gene activities, it is likely to believe that they are involved in many of these key processes. Similar findings were observed by KEGG analysis showing that distinct biological processes and significant pathways were associated with predicted targets of miRNAs exhibiting significantly different levels of expression in LS8 cells during one day cycle of 24 hours. In accordance with previous findings (29), differentially expressed miRNAs likely play a role in specific activities such as ion transport, endocytosis, and apoptosis (Figures $3 \& 4$ ). However, the prediction of miRNA targets using the current algorithms implemented in computational methods always results in a large number of false signals that do not reflect the situation in vivo; therefore, the predicted miRNA targets must be validated experimentally (54). The weakness of this study is that such a high number of predicted miRNA targets are impractical for further understanding of their function, therefore, in our future studies we are planning to conduct a parallel alignment analysis between the list of predicted targets and the list of stage-specific genes identified by total genome sequencing. However, in this study we have focused on mapping the expression and oscillation of miRNAs in LS8 cells during oneday cycle of 24 hours. 
Collectively, our results show that miRNAs are highly expressed in LS8 cells and that many of them oscillate during one-day cycle of 24 hours. Provided that the ameloblast-like LS 8 cell line`s miRNA expression profile is representative of ameloblasts in vivo, it is reasonable to believe that miRNAs represent an essential mechanism influencing gene activity and circadian rhythm. Furthermore, our study highlights the complexity of miRNA regulation in ameloblasts and opens novel directions for future research. Our laboratory will further focus on the miRNA expression and functional experiments in LS8 cells, in order to gain deeper insights into the regulating functions of specific miRNAs. Further studies are required to clarify the mechanisms of the miRNA regulation of transcripts related to amelogenesis.

\section{Acknowledgements}

Funding was obtained from the Department of Oral Biology (University of Oslo) and the Department of Medical Biochemistry (Oslo University Hospital).

\section{Conflict of Interest}

The authors declare that they have no competing interests. 


\section{Legends to Figures}

\section{Figure 1. Length Distribution of Small RNAs}

The clean reads of small RNAs were clustered into unique sequences, and the length of RNAs were identified. The plotted data represent average sequence length distributions for all samples combined (T0 (A, B, C), T6 (A, B, C), T12 (A, B, C), and T18 (A, B, C)). The reads of 21-24 nt accounted for $77.5 \%$ of small RNAs, with $35.81 \%$ of reads exhibiting 22 nt length.

\section{Figure 2. Venn Diagram Revealing Uniquely and Commonly Expressed MicroRNAs}

In total, 191 known miRNAs exhibited significantly (P-value $<0.01)$ different levels of expression across three time-points investigated (T6, T12, and T18) compared to T0. Number of miRNAs that are uniquely and commonly expressed within each time-point is presented in the figure.

\section{Figure 3. Verification of Expression Analysis with qRT-PCR}

Expression patterns of seven selected miRNAs verified with qPCR were in agreement with results from Next-Generation Sequencing (NGS). T0 was used as a reference for other timepoints and each time-point represent the average of samples in triplicate. Both NGS and qPCR showed that mmu-miR-423-3p may serve as housekeeping/reference miRNA. 
Figure 4. GO Analysis of Predicted Targets that were Associated with Differentially Expressed MicroRNAs

The numbers on the right-hand side of each column indicate the number of predicted genes associated with each category. BP: biological processes, CC: cellular components, MP: molecular functions.

\section{Figure 5. Pathway Analysis based on the KEGG Database}

Pathway analysis of predicted genes in biological system according to KEGG is presented in the figure. The numbers on the right-hand side of each column indicate the number of predicted genes associated with each category. A, B, C: Pathway analysis of predicted genes associated with miRNAs that were differentially expressed at T6, T12, and T18 compared to T0, respectively. 
Table 1. Detection of Known and Novel MicroRNAs in LS8 Cells

\begin{tabular}{|l|c|c|c|}
\hline Sample & Known miRNAs $(\boldsymbol{\%})$ & Novel miRNAs $(\%)$ & Total \\
\hline Total & $\mathbf{1 3 3 0}(\mathbf{9 0 . 4 1})$ & $\mathbf{1 3 9}(\mathbf{9 . 5 9})$ & $\mathbf{1 4 7 1}$ \\
\hline T0-A & $965(91.64)$ & $88(8.36)$ & 1053 \\
\hline T0-B & $985(91.46)$ & $92(8.54)$ & 1077 \\
\hline T0-C & $973(91.62)$ & $89(8.38)$ & 1062 \\
\hline T6-A & $968(90.81)$ & $98(9.19)$ & 1066 \\
\hline T6-B & $952(91.27)$ & $91(8.73)$ & 1043 \\
\hline T6-C & $976(91.30)$ & $93(8.70)$ & 1069 \\
\hline T12-A & $992(90.93)$ & $99(9.07)$ & 1091 \\
\hline T12-B & $997(91.55)$ & $92(8.45)$ & 1089 \\
\hline T12-C & $973(91.97)$ & $85(8.03)$ & 1063 \\
\hline T18-A & $965(90.78)$ & $98(9.22)$ & 1082 \\
\hline T18-B & $991(91.59)$ & $91(8.41)$ & 1066 \\
\hline T18-C & $974(91.37)$ & $92(8.63)$ & \\
\hline
\end{tabular}

Number of detected known and novel miRNAs at each time-point investigated, including all triplicate samples, is presented in the table. 
Table 2. Differentially Expressed MicroRNAs at T6 compared to T0

\begin{tabular}{|c|c|c|c|c|c|}
\hline $\begin{array}{c}\text { Down-regulated } \\
\text { miRNAs (T6 vs. T0) }\end{array}$ & $\begin{array}{l}\text { Fold change } \\
\quad(\log 2)\end{array}$ & P-value & $\begin{array}{c}\text { Up-regulated } \\
\text { miRNAs (T6 vs. T0) }\end{array}$ & $\begin{array}{l}\text { Fold change } \\
\quad(\log 2)\end{array}$ & P-value \\
\hline mmu-let-7a-1-3p & -0.47 & $4.92 \mathrm{e}-07$ & mmu-miR-101a-3p & 0.22052 & 0.0001 \\
\hline mmu-let-7a-2-3p & -0.43865 & 0.0014 & mmu-miR-101b-3p & 0.30951 & 0.0033 \\
\hline mmu-let-7b-3p & -0.65133 & $5.67 \mathrm{e}-14$ & mmu-miR-103-3p & 0.36199 & $1.25 \mathrm{e}-06$ \\
\hline$m m u-l e t-7 f-1-3 p$ & -0.33711 & 0.0051 & mmu-miR-107-3p & 0.31384 & 0.0005 \\
\hline mmu-miR-125b-1-3p & -0.82452 & $1.45 \mathrm{e}-20$ & mmu-miR-125b-2-3p & 0.35167 & 0.0039 \\
\hline mmu-miR-126a-3p & -0.46818 & 0.0109 & mmu-miR-140-3p & 0.50048 & $1.75 \mathrm{e}-07$ \\
\hline mmu-miR-129-5p & -0.57465 & $4.19 \mathrm{e}-07$ & mmu-miR-148b-3p & 0.17145 & 0.0059 \\
\hline mmu-miR-129b-3p & -0.57182 & $3.47 \mathrm{e}-07$ & mmu-miR-152-3p & 0.33952 & $2.69 \mathrm{e}-06$ \\
\hline mmu-miR-145a-3p & -0.51539 & $1.03 \mathrm{e}-08$ & mmu-miR-15b-5p & 0.26248 & 0.0012 \\
\hline mmu-miR-145a-5p & -0.18273 & $1.28 \mathrm{e}-08$ & mmu-miR-17-5p & 0.26965 & 0.0048 \\
\hline mmu-miR-148a-3p & -0.77777 & 0.0105 & mmu-miR-181b-1-3p & 0.31858 & 0.0058 \\
\hline mmu-miR-155-3p & -0.62048 & $1.27 \mathrm{e}-06$ & mmu-miR-194-5p & 0.33398 & 0.0042 \\
\hline mmu-miR-16-1-3p & -0.23913 & 0.0016 & mmu-miR-195a-5p & 0.17097 & 0.0012 \\
\hline mmu-miR-181a-1-3p & -0.29094 & 0.0025 & mmu-miR-199a-3p & 0.17796 & 0.0004 \\
\hline mmu-miR-182-3p & -0.80918 & $1.42 \mathrm{e}-28$ & mmu-miR-20a-5p & 0.27019 & 0.0062 \\
\hline mmu-miR-193a-5p & -0.44497 & $1.77 \mathrm{e}-05$ & mmu-miR-210-5p & 0.33442 & 0.0068 \\
\hline mmu-miR-193b-3p & -0.40658 & 0.0061 & mmu-miR-23a-3p & 0.41497 & $4.79 \mathrm{e}-21$ \\
\hline mmu-miR-195a-3p & -0.21712 & $6.85 \mathrm{e}-05$ & mmu-miR-23b-3p & 0.49357 & $7.11 \mathrm{e}-12$ \\
\hline mmu-miR-1981-5p & -0.34186 & 0.0012 & mmu-miR-3068-3p & 0.44947 & 0.0002 \\
\hline mmu-miR-212-5p & -0.34072 & 0.0033 & mmu-miR-30b-5p & 0.19646 & 0.0026 \\
\hline mmu-miR-21a-3p & -0.40162 & $8.56 \mathrm{e}-07$ & mmu-miR-322-3p & 0.26652 & 0.0059 \\
\hline mmu-miR-221-5p & -0.42625 & $9.21 \mathrm{e}-10$ & mmu-miR-324-5p & 0.27399 & 0.0088 \\
\hline mmu-miR-222-5p & -0.46206 & $4.52 \mathrm{e}-11$ & mmu-miR-345-3p & 0.34393 & $3.64 \mathrm{e}-08$ \\
\hline mmu-miR-25-5p & -0.34687 & 0.0015 & mmu-miR-361-5p & 0.20163 & 0.0041 \\
\hline mmu-miR-27a-5p & -0.2728 & $8.10 \mathrm{e}-08$ & mmu-miR-362-3p & 0.36153 & 0.0041 \\
\hline mmu-miR-296-3p & -0.13532 & 0.0002 & mmu-miR-378b & 0.32613 & 0.0014 \\
\hline mmu-miR-29b-1-5p & -0.60327 & $3.11 \mathrm{e}-06$ & mmu-miR-378c & 0.25899 & 0.0077 \\
\hline mmu-miR-30b-3p & -0.25135 & 0.0078 & mmu-miR-450b-5p & 0.56604 & $5.21 \mathrm{e}-12$ \\
\hline mmu-miR-31-3p & -0.42184 & 0.0001 & mmu-miR-455-3p & 0.35695 & 0.0002 \\
\hline mmu-miR-342-5p & -0.37906 & 0.0072 & mmu-miR-532-3p & 0.36165 & 0.0010 \\
\hline mmu-miR-3473b & -0.5091 & 0.0023 & mmu-miR-671-3p & 0.40715 & 0.0002 \\
\hline mmu-miR-365-2-5p & -0.71973 & $3.74 \mathrm{e}-11$ & mmu-miR-872-5p & 0.11617 & 0.0101 \\
\hline \multirow[t]{2}{*}{ mmu-miR-423-5p } & -0.25178 & 0.0005 & mmu-miR-92a-3p & 0.26373 & 0.0072 \\
\hline & & & mmu-miR-93-5p & 0.16943 & 0.0021 \\
\hline
\end{tabular}


The differential expression analysis showed that 67 known miRNAs exhibited significantly (Pvalue $<0.01$ ) different levels of expression at T6 compared to T0, with 33 miRNAs being downregulated and 34 up-regulated. 
Table 3. Differentially Expressed MicroRNAs at T12 compared to T0

\begin{tabular}{|l|c|c|l|c|c|}
\hline $\begin{array}{c}\text { Down-regulated } \\
\text { miRNAs (T12 vs. T0) }\end{array}$ & $\begin{array}{c}\text { Fold change } \\
\text { (Log2) }\end{array}$ & P-value & $\begin{array}{c}\text { Up-regulated } \\
\text { miRNAs (T12 vs. T0) }\end{array}$ & $\begin{array}{c}\text { Fold change } \\
\text { (Log2) }\end{array}$ & P-value \\
\hline mmu-miR-181b-1-3p & -1.0328 & 0.0002 & mmu-miR-152-5p & 0.63045 & $2.66 \mathrm{e}-05$ \\
\hline mmu-miR-6948-5p & -2.1795 & 0.0001 & mmu-miR-370-3p & 1.745 & $3.17 \mathrm{e}-06$ \\
\hline & & & mmu-miR-7063-5p & 2.0212 & 0.0001 \\
\hline
\end{tabular}

The differential expression analysis showed that 5 known miRNAs exhibited significantly (Pvalue $<0.01$ ) different levels of expression at T12 compared to T0, with 2 miRNAs being downregulated and 3 up-regulated. 
Table 4. Differentially Expressed MicroRNAs at T18 compared to T0

\begin{tabular}{|c|c|c|c|c|c|}
\hline $\begin{array}{c}\text { Down-regulated } \\
\text { miRNAs (T18 vs. T0) }\end{array}$ & $\begin{array}{l}\text { Fold change } \\
\quad(\log 2)\end{array}$ & P-value & $\begin{array}{c}\text { Up-regulated } \\
\text { miRNAs (T18 vs. T0) }\end{array}$ & $\begin{array}{l}\text { Fold change } \\
\quad(\log 2)\end{array}$ & P-value \\
\hline mmu-let-7d-5p & -0.29513 & 0.0002 & mmu-let-7d-3p & 0.18781 & 0.0001 \\
\hline mmu-let-7g-5p & -0.2639 & 0.0077 & mmu-let-7f-1-3p & 0.42039 & 0.0003 \\
\hline mmu-let-7i-5p & -0.1574 & 0.0096 & mmu-miR-100-3p & 0.31362 & 0.0014 \\
\hline mmu-let-7j & -3.8908 & $3.96 \mathrm{e}-134$ & mmu-miR-100-5p & 0.24995 & 0.0115 \\
\hline mmu-miR-101a-3p & -0.48562 & $1.59 \mathrm{e}-05$ & mmu-miR-10a-5p & 0.19845 & 0.0027 \\
\hline mmu-miR-101b-3p & -0.53454 & $6.39 \mathrm{e}-05$ & mmu-miR-10b-5p & 0.18502 & 0.0154 \\
\hline mmu-miR-106b-3p & -0.22067 & $3.79 \mathrm{e}-06$ & mmu-miR-125b-2-3p & 0.374 & 0.0021 \\
\hline mmu-miR-125b-1-3p & -0.23407 & 0.0065 & mmu-miR-134-5p & 0.52758 & 0.0080 \\
\hline mmu-miR-127-3p & -2.9228 & $2.41 \mathrm{e}-15$ & mmu-miR-135b-5p & 0.63749 & $4.49 \mathrm{e}-07$ \\
\hline mmu-miR-128-1-5p & -0.47934 & 0.0009 & mmu-miR-140-5p & 0.33312 & $2.33 \mathrm{e}-10$ \\
\hline mmu-miR-129-5p & -0.51081 & $1.35 \mathrm{e}-06$ & mmu-miR-148b-3p & 0.19168 & 0.0040 \\
\hline mmu-miR-129b-3p & -0.50973 & $2.21 \mathrm{e}-06$ & mmu-miR-148b-5p & 0.18991 & 0.0165 \\
\hline mmu-miR-130a-3p & -0.38959 & 0.0015 & mmu-miR-149-5p & 0.6477 & $1.23 \mathrm{e}-24$ \\
\hline mmu-miR-130b-3p & -0.5289 & 0.0018 & mmu-miR-151-3p & 0.36737 & 0.0013 \\
\hline mmu-miR-140-3p & -0.5698 & 0.0033 & mmu-miR-152-5p & 0.4146 & 0.0003 \\
\hline mmu-miR-143-3p & -0.17871 & 0.0036 & mmu-miR-1843b-3p & 0.41875 & 0.0055 \\
\hline mmu-miR-143-5p & -0.26619 & 0.0002 & mmu-miR-1934-5p & 0.39455 & 0.0113 \\
\hline mmu-miR-145a-3p & -0.41767 & $5.16 \mathrm{e}-05$ & mmu-miR-1941-3p & 0.41674 & 0.0004 \\
\hline mmu-miR-146a-5p & -0.39343 & & mmu-miR-195a-3p & 0.28372 & 0.0045 \\
\hline mmu-miR-149-3p & -0.96935 & 0.0003 & mmu-miR-1964-3p & 0.42899 & 0.0016 \\
\hline mmu-miR-152-3p & -0.57672 & 0.0048 & mmu-miR-1981-5p & 0.53722 & $2.75 e-05$ \\
\hline mmu-miR-155-3p & -0.48626 & 0.0001 & mmu-miR-199a-5p & 0.43697 & $1.11 \mathrm{e}-07$ \\
\hline mmu-miR-15a-5p & -0.42121 & 0.0026 & mmu-miR-199b-5p & 0.31528 & 0.0011 \\
\hline mmu-miR-15b-5p & -0.38915 & & mmu-miR-200c-3p & 0.82692 & $1.35 \mathrm{e}-06$ \\
\hline mmu-miR-17-3p & -0.51783 & 0.0019 & mmu-miR-210-3p & 0.35301 & 0.0005 \\
\hline mmu-miR-17-5p & -0.47433 & 0.0106 & mmu-miR-210-5p & 1.1344 & $3.09 \mathrm{e}-25$ \\
\hline mmu-miR-181b-1-3p & -1.4585 & $4.44 \mathrm{e}-08$ & mmu-miR-214-5p & 0.38959 & 0.0003 \\
\hline mmu-miR-182-3p & -0.73476 & $9.69 \mathrm{e}-25$ & mmu-miR-218-5p & 0.34113 & 0.0029 \\
\hline mmu-miR-183-3p & -0.71284 & 0.0002 & mmu-miR-219a-1-3p & 0.23668 & 0.0069 \\
\hline mmu-miR-183-5p & -0.31969 & 0.0015 & mmu-miR-21a-5p & 0.16107 & 0.0108 \\
\hline mmu-miR-1839-5p & -0.49052 & 0.0002 & mmu-miR-24-3p & 0.26143 & 0.0007 \\
\hline mmu-miR-192-5p & -0.10942 & 0.0166 & mmu-miR-26a-1-3p & 0.98296 & $8.49 \mathrm{e}-07$ \\
\hline mmu-miR-193a-5p & -0.97971 & $8.28 \mathrm{e}-08$ & mmu-miR-26a-2-3p & 0.61549 & $5.72 \mathrm{e}-16$ \\
\hline mmu-miR-19a-3p & -0.70232 & 0.0015 & mmu-miR-27a-3p & 0.37332 & 0.0045 \\
\hline mmu-miR-19b-3p & -0.81359 & 0.0004 & mmu-miR-29a-3p & 0.18267 & 0.0109 \\
\hline mmu-miR-217-5p & -1.2885 & $3.44 \mathrm{e}-09$ & mmu-miR-29c-5p & 0.47898 & 0.0004 \\
\hline mmu-miR-221-5p & -0.63841 & $2.29 \mathrm{e}-07$ & mmu-miR-301a-5p & 0.2526 & 0.0103 \\
\hline mmu-miR-222-5p & -0.30348 & $9.12 \mathrm{e}-06$ & mmu-miR-3074-5p & 0.26406 & 0.0006 \\
\hline mmu-miR-23a-5p & -1.103 & $9.92 \mathrm{e}-10$ & mmu-miR-30d-3p & 0.31194 & 0.0169 \\
\hline mmu-miR-23b-5p & -0.78553 & $1.29 \mathrm{e}-06$ & mmu-miR-32-5p & 0.42086 & 0.0001 \\
\hline mmu-miR-25-5p & -0.7331 & $9.24 \mathrm{e}-08$ & mmu-miR-328-3p & 0.53336 & $7.69 \mathrm{e}-12$ \\
\hline mmu-miR-27a-5p & -0.86276 & $6.81 \mathrm{e}-88$ & mmu-miR-339-5p & 0.29331 & 0.0002 \\
\hline mmu-miR-27b-5p & -0.77552 & $5.46 \mathrm{e}-10$ & mmu-miR-340-3p & 0.40119 & $5.84 \mathrm{e}-08$ \\
\hline mmu-miR-28a-3p & -0.47515 & $2.82 \mathrm{e}-17$ & mmu-miR-344-3p & 0.33961 & 0.0005 \\
\hline
\end{tabular}




\begin{tabular}{|c|c|c|c|c|c|}
\hline mmu-miR-28a-5p & -0.32257 & 0.0005 & mmu-miR-34b-3p & 0.22922 & $1.85 \mathrm{e}-19$ \\
\hline mmu-miR-296-3p & -0.22888 & $3.24 \mathrm{e}-05$ & mmu-miR-365-1-5p & 0.50242 & 0.0005 \\
\hline mmu-miR-29b-1-5p & -0.98934 & $4.26 \mathrm{e}-06$ & mmu-miR-370-3p & 1.1547 & $2.29 \mathrm{e}-07$ \\
\hline mmu-miR-3057-5p & -0.47255 & 0.0025 & mmu-miR-374b-5p & 0.29442 & $1.79 \mathrm{e}-05$ \\
\hline mmu-miR-3064-5p & -0.62596 & 0.0132 & mmu-miR-374c-3p & 0.29546 & $1.47 \mathrm{e}-05$ \\
\hline mmu-miR-3066-5p & -0.23454 & 0.0009 & mmu-miR-378a-5p & 0.36127 & $3.79 \mathrm{e}-07$ \\
\hline mmu-miR-3091-3p & -0.69825 & 0.0085 & mmu-miR-409-3p & 0.60083 & 0.0003 \\
\hline mmu-miR-30a-3p & -0.16288 & $9.43 \mathrm{e}-07$ & mmu-miR-411-5p & 0.7272 & 0.0004 \\
\hline mmu-miR-30a-5p & -0.64953 & $3.23 \mathrm{e}-06$ & mmu-miR-425-5p & 0.5578 & $2.74 \mathrm{e}-07$ \\
\hline mmu-miR-30b-3p & -0.23429 & 0.0044 & mmu-miR-450a-5p & 0.23446 & 0.0002 \\
\hline mmu-miR-30b-5p & -0.22881 & 0.0061 & mmu-miR-450b-5p & 1.0932 & $4.51 \mathrm{e}-27$ \\
\hline mmu-miR-30c-2-3p & -0.52232 & $4.03 \mathrm{e}-05$ & mmu-miR-455-5p & 0.31188 & 0.0035 \\
\hline mmu-miR-30e-5p & -0.40808 & 0.0083 & mmu-miR-466i-5p & 0.82536 & 0.0028 \\
\hline mmu-miR-31-5p & -0.4096 & 0.0088 & mmu-miR-467a-5p & 0.39011 & 0.0013 \\
\hline mmu-miR-340-5p & -0.51124 & $4.99 \mathrm{e}-07$ & mmu-miR-467b-5p & 0.39011 & 0.0013 \\
\hline mmu-miR-342-5p & -0.34876 & 0.0014 & mmu-miR-467d-5p & 0.60421 & 0.0045 \\
\hline mmu-miR-344d-3p & -0.1597 & 0.0019 & mmu-miR-484 & 0.26291 & 0.0028 \\
\hline mmu-miR-345-3p & -0.20415 & 0.0121 & mmu-miR-497a-5p & 0.29202 & $7.22 \mathrm{e}-10$ \\
\hline mmu-miR-3535 & -0.56756 & $1.92 \mathrm{e}-07$ & mmu-miR-497b & 0.58016 & 0.0143 \\
\hline mmu-miR-365-3p & -0.4317 & 0.0006 & mmu-miR-503-3p & 0.31873 & 0.0004 \\
\hline mmu-miR-378b & -0.62469 & $6.16 \mathrm{e}-05$ & mmu-miR-5113 & 0.79001 & 0.0035 \\
\hline mmu-miR-378c & -0.7766 & $1.19 \mathrm{e}-05$ & mmu-miR-598-3p & 0.42725 & 0.0002 \\
\hline mmu-miR-450b-3p & -0.58618 & 0.0015 & mmu-miR-615-5p & 0.87597 & $2.01 \mathrm{e}-07$ \\
\hline mmu-miR-466d-3p & -0.39181 & 0.0115 & mmu-miR-664-5p & 0.26157 & 0.0121 \\
\hline mmu-miR-491-5p & -0.45497 & 0.0103 & mmu-miR-671-3p & 0.40533 & 0.0003 \\
\hline mmu-miR-5099 & -0.47571 & 0.0082 & mmu-miR-671-5p & 0.73236 & $1.57 \mathrm{e}-17$ \\
\hline mmu-miR-542-3p & -0.61854 & 0.0025 & mmu-miR-674-3p & 0.26727 & 0.0007 \\
\hline mmu-miR-6539 & -0.64119 & $3.23 \mathrm{e}-06$ & mmu-miR-690 & 0.90622 & $3.09 \mathrm{e}-08$ \\
\hline mmu-miR-669c-5p & -0.28985 & 0.0047 & mmu-miR-700-5p & 0.41043 & 0.0024 \\
\hline mmu-miR-708-5p & -0.69537 & $3.32 \mathrm{e}-05$ & mmu-miR-93-3p & 0.2749 & 0.0036 \\
\hline mmu-miR-744-5p & -0.36899 & 0.0031 & mmu-miR-96-5p & 0.32808 & 0.0088 \\
\hline mmu-miR-7650-5p & -0.60991 & 0.0067 & mmu-miR-98-3p & 0.46368 & 0.0077 \\
\hline mmu-miR-9-3p & -0.4342 & 0.0018 & mmu-miR-99a-5p & 0.43962 & 0.0047 \\
\hline mmu-miR-96-3p & -1.0833 & $5.23 e-05$ & mmu-miR-99b-5p & 0.23721 & 0.0015 \\
\hline
\end{tabular}

The differential expression analysis showed that 156 known miRNAs exhibited significantly (P-value $<0.01$ ) different levels of expression at T18 compared to T0, with 78 miRNAs being down-regulated and 78 up-regulated. 
Supplementary material 1. Summary of Small RNA Sequencing Data

\begin{tabular}{|l|l|l|l|l|l|l|}
\hline Sample & Raw Reads & Bases & Error rate & Q20 & Q30 & GC content \\
\hline T0-A & 20917106 & $1.046 \mathrm{G}$ & $0.01 \%$ & $97.81 \%$ & $95.68 \%$ & $49.29 \%$ \\
\hline T0-B & 21934441 & $1.097 \mathrm{G}$ & $0.01 \%$ & $97.78 \%$ & $95.64 \%$ & $49.43 \%$ \\
\hline T0-C & 21276251 & $1.064 \mathrm{G}$ & $0.01 \%$ & $97.81 \%$ & $95.67 \%$ & $49.28 \%$ \\
\hline T6-A & 21937668 & $1.097 \mathrm{G}$ & $0.01 \%$ & $97.84 \%$ & $95.73 \%$ & $49.43 \%$ \\
\hline T6-B & 20870682 & $1.044 \mathrm{G}$ & $0.01 \%$ & $97.91 \%$ & $95.81 \%$ & $49.75 \%$ \\
\hline T6-C & 23283385 & $1.164 \mathrm{G}$ & $0.01 \%$ & $97.99 \%$ & $95.94 \%$ & $49.41 \%$ \\
\hline T12-A & 20273866 & $1.014 \mathrm{G}$ & $0.01 \%$ & $97.88 \%$ & $95.78 \%$ & $49.33 \%$ \\
\hline T12-B & 20313604 & $1.016 \mathrm{G}$ & $0.01 \%$ & $97.32 \%$ & $94.59 \%$ & $49.59 \%$ \\
\hline T12-C & 21045646 & $1.052 \mathrm{G}$ & $0.01 \%$ & $97.37 \%$ & $94.72 \%$ & $50.35 \%$ \\
\hline T18-A & 21282650 & $1.064 \mathrm{G}$ & $0.01 \%$ & $97.89 \%$ & $95.67 \%$ & $49.55 \%$ \\
\hline T18-B & 22098881 & $1.105 \mathrm{G}$ & $0.01 \%$ & $98.02 \%$ & $95.93 \%$ & $49.48 \%$ \\
\hline T18-C & 21376613 & $1.069 \mathrm{G}$ & $0.01 \%$ & $97.53 \%$ & $95.13 \%$ & $49.43 \%$ \\
\hline
\end{tabular}

The overview of the original sequence data with raw reads are presented in the table. Bases: sequence multiplied the length of the sequence and converted to the unit of G. Error rate: sequencing error rate. Q20: Phred values greater than 20 base percentage. Q30: Phred values greater than 30 base percentage. GC content: The G base and C base accounted for the overall base. 
Supplementary material 2. Clean Process Overview

\begin{tabular}{|l|c|c|c|c|c|c|}
\hline Sample & $\begin{array}{c}\text { Total } \\
\text { reads }\end{array}$ & $\begin{array}{c}\text { High } \\
\text { quality }\end{array}$ & $\begin{array}{c}\text { Adapter 5' } \\
\text { contaminants }\end{array}$ & $\begin{array}{c}\text { Adapter 3'null } \\
\text { / insert null }\end{array}$ & $\begin{array}{c}\text { Poly } \\
\text { A/T/G/C }\end{array}$ & Clean reads (\%) \\
\hline T0-A & 20917106 & $99.76 \%$ & $746(0.00 \%)$ & $2.50 \%$ & $0.44 \%$ & $20252715(96.82)$ \\
\hline T0-B & 21934441 & $99.72 \%$ & $1731(0.01 \%)$ & $3.22 \%$ & $0.39 \%$ & $21077412(96.09)$ \\
\hline T0-C & 21276251 & $99.73 \%$ & $1140(0.01 \%)$ & $3.89 \%$ & $0.35 \%$ & $20314535(95.48)$ \\
\hline T6-A & 21937668 & $99.71 \%$ & $1102(0.01 \%)$ & $1.50 \%$ & $0.30 \%$ & $21477122(97.90)$ \\
\hline T6-B & 20870682 & $99.78 \%$ & $872(0.00 \%)$ & $1.55 \%$ & $0.34 \%$ & $20427312(97.88)$ \\
\hline T6-C & 23283385 & $99.77 \%$ & $757(0.00 \%)$ & $3.28 \%$ & $0.29 \%$ & $22397187(96.19)$ \\
\hline T12-A & 20273866 & $99.77 \%$ & $862(0.00 \%)$ & $1.77 \%$ & $0.31 \%$ & $19803177(97.68)$ \\
\hline T12-B & 20313604 & $99.73 \%$ & $925(0.00 \%)$ & $1.46 \%$ & $0.36 \%$ & $19886249(97.90)$ \\
\hline T12-C & 21045646 & $99.72 \%$ & $1945(0.01 \%)$ & $2.76 \%$ & $0.44 \%$ & $20310612(96.51)$ \\
\hline T18-A & 21282650 & $99.78 \%$ & $834(0.00 \%)$ & $2.41 \%$ & $0.31 \%$ & $20655205(97.05)$ \\
\hline T18-B & 22098881 & $99.78 \%$ & $1066(0.00 \%)$ & $1.41 \%$ & $0.21 \%$ & $21690211(98.15)$ \\
\hline T18-C & 21376613 & $99.60 \%$ & $1257(0.01 \%)$ & $1.38 \%$ & $0.23 \%$ & $20942795(97.97)$ \\
\hline
\end{tabular}

The overview of cleaning process from raw reads to clean reads is presented in the table.

Initially, the reads containing poly $\mathrm{Ns}(\mathrm{N}>10 \%)$ are removed resulting in high quality reads.

Subsequently, reads with the 5'-adapter contaminants, reads without the 3 '-adapter or insert tags, and reads with poly $\mathrm{A} / \mathrm{T} / \mathrm{G} / \mathrm{C}$ are removed. The final remaining reads are defined as clean. 


\section{Supplementary material 3. Expression of Novel MicroRNAs in LS8 cells}

\begin{tabular}{|c|c|c|c|c|c|c|c|}
\hline \multirow[b]{2}{*}{ miRNA } & \multirow[b]{2}{*}{ miRNA sequence } & \multicolumn{2}{|c|}{ T6 vs. T0 } & \multicolumn{2}{|c|}{ T12 vs. T0 } & \multicolumn{2}{|c|}{ T18 vs. T0 } \\
\hline & & $\begin{array}{l}\text { Fold } \\
\text { change } \\
(\log 2)\end{array}$ & P-value & $\begin{array}{c}\text { Fold } \\
\text { change } \\
(\log 2)\end{array}$ & P-value & $\begin{array}{l}\text { Fold } \\
\text { change } \\
(\log 2)\end{array}$ & P-value \\
\hline novel_1 & uggugggugcauauguuu & 0,085109 & 0,64343 & $-0,55865$ & 0,17139 & $-0,25327$ & 0,21817 \\
\hline novel_10 & ugccucuggaagguuuggcagg & & & 0,87491 & 0,12335 & & \\
\hline novel_100 & acagcuccucucucucucugaagu & & & $-0,04555$ & 0,93554 & & \\
\hline novel_101 & acccggcuaggccugcucugucu & & & 0,17988 & 0,73644 & & \\
\hline novel_106 & agggggugggggguuugga & & & 0,80702 & 0,098776 & & \\
\hline novel_11 & gaaggugggugcuaagggcuga & & & 1,0196 & 0,069265 & & \\
\hline novel_111 & aagcuggguugagagggca & & & 1,3484 & 0,018627 & & \\
\hline novel_112 & uggcuggagauugacgcugguu & & & 0,86982 & 0,071845 & & \\
\hline novel_117 & ccuagaaaaauacuggaugaau & & & $-0,32504$ & 0,56779 & & \\
\hline novel_12 & uuugucauccagauccaccagc & & & $-0,63886$ & 0,26004 & & \\
\hline novel_123 & agaagaaggcuguauugcaaga & & & 0,82307 & 0,11317 & & \\
\hline novel_128 & gggggguggugugguggug & & & 0,12125 & 0,82599 & & \\
\hline novel_134 & uaacgugugaguguguguuuu & & & $-0,32176$ & 0,5743 & & \\
\hline novel_138 & aaagacuggagagguggcuc & & & 0,56635 & 0,27423 & & \\
\hline novel_140 & uguaugacuuuaaacugcaga & & & 0,60264 & 0,29101 & & \\
\hline novel_144 & aggaagagucaggacugg & & & 1,8983 & $0,00091 *$ & & \\
\hline novel_146 & uggaaggacugagcaaguuucu & & & 0,20462 & 0,71258 & & \\
\hline novel_15 & uuggggcagagcacuugguuggu & & & 1,8658 & $0,00108 *$ & & \\
\hline novel_3 & accgggugcuguaggcua & 0,33707 & 0,36109 & $-0,90912$ & 0,029502 & $-0,42526$ & 0,26351 \\
\hline novel_4 & uucauuguugauuuucgguggga & & & $-0,16375$ & 0,73269 & & \\
\hline novel_40 & cggcgcccggagcucuggg & & & 0,66242 & 0,24656 & & \\
\hline novel_44 & ucgggcuggggacggaggggga & & & 1,1896 & 0,03791 & & \\
\hline novel_5 & ugccggcggacggcugggaagaga & & & 0,79965 & 0,14015 & & \\
\hline novel_50 & caaccaugucuucuuucccagc & & & 0,65454 & 0,18219 & & \\
\hline novel_52 & ucgugauggaucugucugagcc & & & 0,62866 & 0,22067 & & \\
\hline novel_53 & ugggcccugacucaugcuccaca & & & $-0,48873$ & 0,33082 & & \\
\hline novel_56 & uugggccucagccaaggacugg & & & 0,48151 & 0,39564 & & \\
\hline novel_58 & ucaguggagacugggaagugaga & & & 1,209 & 0,034864 & & \\
\hline novel_59 & uuaggaaaacugaaacuacag & & & 0,63162 & 0,26946 & & \\
\hline novel_6 & uggcucagugguacuuacauaua & & & $-0,57087$ & 0,30598 & & \\
\hline novel_62 & uugugucugucuggcucccagu & & & 0,28284 & 0,42995 & & \\
\hline novel_68 & gcggguugggcugggugga & & & 0,83644 & 0,13012 & & \\
\hline novel_69 & ucgugucuuguguugcagccgga & & & 0,71026 & 0,054344 & 0,55364 & 0,03921 \\
\hline novel_7 & uucucgacgggcugaccugauc & & & 0,97658 & 0,055302 & & \\
\hline novel_74 & uccugcccccuuucccuguaga & & & $-0,13283$ & 0,78842 & & \\
\hline novel_79 & aauuaggcucuggaucuguga & & & 0,56463 & 0,019192 & 0,31605 & 0,078089 \\
\hline novel_8 & cagaaguuugaacaaaccuuuu & & & $-0,11488$ & 0,8179 & & \\
\hline novel_80 & caccggucugcucuccuccgagu & & & 0,74044 & 0,14621 & & \\
\hline novel_81 & ugauauagccaagcccgacugua & & & $-0,7801$ & 0,14442 & & \\
\hline novel_86 & ucucuuguagguguggaccacu & & & $-0,48629$ & 0,21338 & & \\
\hline
\end{tabular}




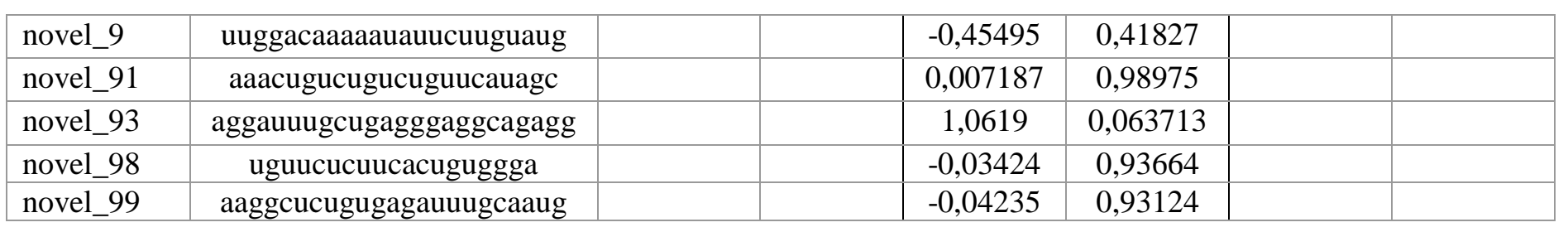

After removing 94 novel miRNAs that were detected with low levels of expression (read count $<5$ across all time-points), 45 miRNAs remained. These novel miRNAs, including their sequences, are presented in the table, with their expression at different time-points compared to T0. * Significantly different expression $(\mathrm{P}$-value $<0.01)$ 


\section{References}

1. Schour I, Massler M. Studies in tooth development: the growth patterns of human teeth. J Am Dens Assoc. 1940;27:1178-93.

2. Schour I, Poncher HG. Rate of apposition of enamel and dentin, measured by the effect of acute fluorosis. Am J Dis Child. 1937;54:757-76.

3. Sehic A, Nirvani M, Risnes S. Incremental lines in mouse molar enamel. Archives of oral biology. 2013;58(10):1443-9.

4. Richards J, Gumz ML. Mechanism of the circadian clock in physiology. Am J Physiol Regul Integr Comp Physiol. 2013;304(12):R1053-64.

5. King DP, Takahashi JS. Molecular genetics of circadian rhythms in mammals. Annu Rev Neurosci. 2000;23:713-42.

6. Fu L, Patel M, Bradley A. The molecular clock modiates leptin-regulated bone formation. Cell. 2005;122:803-15.

7. Zvonic S, Ptitsyn AA, Kilroy G. Circadian oscillation of gene expression in murine calvarian bone. Journal of bone and mineral research : the official journal of the American Society for Bone and Mineral Research. 2007;22:357-65.

8. Nirvani M, Khuu C, Utheim TP, Hollingen HS, Amundsen SF, Sand LP, et al. Circadian rhythms and gene expression during mouse molar tooth development. Acta Odontol Scand. 2017;75(2):144-53.

9. Asli NS, Pitulescu ME, Kessel M. MicroRNAs in organogenesis and disease. Curr Mol Med. 2008;8(8):698-710.

10. Ambros V. The function of animal microRNAs. Nature. 2004;431:350-5.

11. Chen R, D'Alessandro M, Lee C. miRNAs are required for generating a time delay critical for the circadian oscillator. Curr Biol. 2013;23(20):1959-68. 
12. Sehic A, Risnes S, Khuu C, Khan QE, Osmundsen H. Effects of in vivo transfection with anti-miR-214 on gene expression in murine molar tooth germ. Physiol Genomics. 2011;43(9):488-98.

13. Sehic A, Risnes S, Khan QE, Khuu C, Osmundsen H. Gene expression and dental enamel structure in developing mouse incisor. European journal of oral sciences. 2010;118(2):118-30.

14. Jevnaker AM, Osmundsen H. MicroRNA expression profiling of the developing murine molar tooth germ and the developing murine submandibular salivary gland. Archives of oral biology. 2008;53(7):629-45.

15. Sehic A, Tulek A, Khuu C, Nirvani M, Sand LP, Utheim TP. Regulatory roles of microRNAs in human dental tissues. Gene. 2017;596:9-18.

16. Risnes S. Growth tracks in dental enamel. J Hum Evol. 1998;35:331-50.

17. Chen LS, Couwenhoven RI, Hsu D, Luo W, Snead ML. Maintenance of amelogenin gene expression by transformed epithelial cells of mouse enamel organ. Archives of oral biology. 1992;37(10):771-8.

18. Sarkar J, Simanian EJ, Tuggy SY, Bartlett JD, Snead ML, Sugiyama T, et al. Comparison of two mouse ameloblast-like cell lines for enamel-specific gene expression. Frontiers in physiology. 2014;5:277.

19. Wang H, Tannukit S, Zhu D, Snead ML, Paine ML. Enamel matrix protein interactions. Journal of bone and mineral research : the official journal of the American Society for Bone and Mineral Research. 2005;20(6):1032-40.

20. Sharma R, Tsuchiya M, Bartlett JD. Fluoride induces endoplasmic reticulum stress and inhibits protein synthesis and secretion. Environmental health perspectives. 2008;116(9):1142-6. 
21. Zhou YL, Lei Y, Snead ML. Functional antagonism between Msx2 and

CCAAT/enhancer-binding protein alpha in regulating the mouse amelogenin gene expression is mediated by protein-protein interaction. The Journal of biological chemistry. 2000;275(37):29066-75.

22. Zhou YL, Snead ML. Identification of CCAAT/enhancer-binding protein alpha as a transactivator of the mouse amelogenin gene. The Journal of biological chemistry. 2000;275(16):12273-80.

23. Xu Y, Zhou YL, Ann DK, MacDougald OA, Shum L, Snead ML. Transcription factor sumoylation and factor YY1 serve to modulate mouse amelogenin gene expression. European journal of oral sciences. 2006;114(Suppl 1):169-77.

24. Xu Y, Zhou YL, Luo W, Zhu QS, Levy D, MacDougald OA. NF-Y and CCAAT/enhancer-binding protein alpha synergistically activate the mouse amelogenin gene. The Journal of biological chemistry. 2006;281:16090-8.

25. Dhamija S, Krebsbach PH. Role of Cbfa1 in ameloblastin gene transcription. The Journal of biological chemistry. 2001;276(37):35159-64.

26. Dhamija S, Liu Y, Yamada Y, Snead ML, Krebsbach PH. Cloning and characterization of the murine ameloblastin promoter. The Journal of biological chemistry. 1999;274(29):20738-43.

27. Kubota K, Lee DH, Tsuchiya M, Young CS, Everett ET, Martinez-Mier EA, et al. Fluoride induces endoplasmic reticulum stress in ameloblasts responsible for dental enamel formation. The Journal of biological chemistry. 2005;280(24):23194-202.

28. Lacruz RS, Hacia JG, Bromage TG, Boyde A, Lei Y, Xu Y, et al. The circadian clock modulates enamel development. Journal of biological rhythms. 2012;27(3):237-45.

29. Yin K, Hacia JG, Zhong Z, Paine ML. Genome-wide analysis of miRNA and mRNA transcriptomes during amelogenesis. BMC Genomics. 2014;15:998. 
30. Yin K, Lin W, Guo J, Sugiyama T, Snead ML, Hacia JG, et al. MiR-153 Regulates Amelogenesis by Targeting Endocytotic and Endosomal/lysosomal Pathways-Novel Insight into the Origins of Enamel Pathologies. Sci Rep. 2017;7:44118.

31. Atanasov AG, Leiser D, Roesselet C, Noti M, Corazza N, Schoonjans K, et al. Cell cycle-dependent regulation of extra-adrenal glucocorticoid synthesis in murine intestinal epithelial cells. FASEB J. 2008;22(12):4117-25.

32. Griffiths-Jones S, Grocock RJ, van Dongen S, Bateman A, Enright AJ. miRBase: microRNA sequences, targets and gene nomenclature. Nucleic Acids Res. 2006;34(Database issue):D140-4.

33. Wen M, Shen Y, Shi S, Tang T. miREvo: an integrative microRNA evolutionary analysis platform for next-generation sequencing experiments. BMC Bioinformatics. 2012;13:140.

34. Friedlander MR, Mackowiak SD, Li N, Chen W, Rajewsky N. miRDeep2 accurately identifies known and hundreds of novel microRNA genes in seven animal clades. Nucleic Acids Res. 2012;40(1):37-52.

35. Storey JD. The positive false discovery rate: A Bayesian interpretation and the qvalue. Ann Stat. 2003;31(6):2013-35.

36. Enright AJ, John B, Gaul U, Tuschl T, Sander C, Marks DS. MicroRNA targets in Drosophila. Genome Biol. 2003;5(1):R1.

37. Young MD, Wakefield MJ, Smyth GK, Oshlack A. Gene ontology analysis for RNAseq: accounting for selection bias. Genome Biol. 2010;11(2):R14.

38. Mao X, Cai T, Olyarchuk JG, Wei L. Automated genome annotation and pathway identification using the KEGG Orthology (KO) as a controlled vocabulary. Bioinformatics. 2005;21(19):3787-93. 
39. Kanehisa M, Araki M, Goto S, Hattori M, Hirakawa M, Itoh M, et al. KEGG for linking genomes to life and the environment. Nucleic Acids Res. 2008;36(Database issue):D480-4.

40. Ewing B, Green P. Base-calling of automated sequencer traces using phred. II. Error probabilities. . Genome Research 1998;8:186-94.

41. Zamore PD, Tuschl T, Sharp PA, Bartel DP. RNAi double-stranded RNA directs the ATP-dependent cleavage of mRNA at 21 to 23 nucleotide intervals. Cell. 2000;101(1):25-33.

42. Cheng HY, Papp JW, Varlamova O, Dziema H, Russell B, Curfman JP, et al. microRNA modulation of circadian-clock period and entrainment. Neuron. 2007;54(5):81329.

43. Mehta P, Goyal S, Wingreen NS. A quantitative comparison of sRNA-based and protein-based gene regulation. Mol Syst Biol. 2008;4:221.

44. Nandi A, Vaz C, Bhattacharya A, Ramaswamy R. miRNA-regulated dynamics in circadian oscillator models. BMC Syst Biol. 2009;3:45.

45. Michon F, Tummers M, Kyyronen M, Frilander MJ, Thesleff I. Tooth morphogenesis and ameloblast differentiation are regulated by micro-RNAs. Dev Biol. 2010;340(2):355-68. 46. Nakata A, Kameda T, Nagai H, Ikegami K, Duan Y, Terada K, et al. Establishment and characterization of a spontaneously immortalized mouse ameloblast-lineage cell line. Biochemical and biophysical research communications. 2003;308(4):834-9.

47. Kawano S, Morotomi T, Toyono T, Nakamura N, Uchida T, Ohishi M, et al. Establishment of dental epithelial cell line (HAT-7) and the cell differentiation dependent on Notch signaling pathway. Connective tissue research. 2002;43(2-3):409-12.

48. Arakaki M, Ishikawa M, Nakamura T, Iwamoto T, Yamada A, Fukumoto E, et al. Role of epithelial-stem cell interactions during dental cell differentiation. The Journal of biological chemistry. 2012;287(13):10590-601. 
49. DenBesten PK, Gao C, Li W, Mathews CH, Gruenert DC. Development and characterization of an SV40 immortalized porcine ameloblast-like cell line. European journal of oral sciences. 1999;107(4):276-81.

50. Zheng L, Papagerakis S, Schnell SD, Hoogerwerf WA, Papagerakis P. Expression of clock proteins in developing tooth. Gene Expr Patterns. 2011;11(3-4):202-6.

51. Git A, Dvinge H, Salmon-Divon M, Osborne M, Kutter C, Hadfield J, et al. Systematic comparison of microarray profiling, real-time PCR, and next-generation sequencing technologies for measuring differential microRNA expression. RNA. 2010;16(5):991-1006.

52. Saus E, Soria V, Escaramis G, Vivarelli F, Crespo JM, Kagerbauer B, et al. Genetic variants and abnormal processing of pre-miR-182, a circadian clock modulator, in major depression patients with late insomnia. Hum Mol Genet. 2010;19(20):4017-25.

53. Yoo JO, Kwak SY, An HJ, Bae IH, Park MJ, Han YH. miR-181b-3p promotes epithelial-mesenchymal transition in breast cancer cells through Snail stabilization by directly targeting YWHAG. Biochimica et biophysica acta. 2016;1863(7 Pt A):1601-11.

54. Ekimler S, Sahin K. Computational Methods for MicroRNA Target Prediction. Genes (Basel). 2014;5(3):671-83. 